\title{
Centro instantâneo de movimento, na avaliação ex-vivo da reconstrução extra-articular fabelo-tibial, após transecção do ligamento cruzado cranial em cães
}

\author{
Instantaneous center of motion following ex-vivo extra-articular stabilization for \\ the cranial cruciate ligament-deficient stifle
}

André Luis Selmi ${ }^{1}$ Bruno Testoni Lins ${ }^{2}$

Glenda Ramalho Barbudo-Selmi ${ }^{1}$ Guiherme Maia Mendes ${ }^{2}$

RESUMO

Este estudo comparou o efeito na biomecânica articular de dois fios de sutura, aço e polipropileno, na estabilização do joelho após transecção do ligamento cruzado cranial de cães, utilizando-se a técnica extraarticular de sutura fabelo-tibial. O centro instantâneo de movimento e o vetor velocidade resultante, foram calculados por meio de análise radiográfica das articulações fêmurtíbio-patelares de doze cães, antes e após a desestabilização e estabilização articular. Todas as articulações apresentavam centro instantâneo e vetor velocidade normais antes da transecção do ligamento. Após a mesma, observou-se o posicionamento anormal do vetor velocidade em onze articulações. Na análise radiográfica posterior à estabilização articular, quatro articulações do grupo nos quais foi empregado o fio de aço continuaram apresentando posicionamento anormal do vetor velocidade, enquanto todas as articulações do grupo em que foi utilizado o fio de polipropileno apresentaram vetor velocidade em posição tangente às superfícies ósseas. Conclui-se que o fio de polipropileno é mais indicado na estabilização extraarticular por manter a biomecânica articular inalterada.

Palavras-chave: cirurgia, biomecânica, ortopedia.

\section{ABSTRACT}

This study evaluated the effect of two suture materials, stainless steel wire and polypropylene, on the stifle joint biomechanics by means of analysis of the instantaneous center of motion after stabilization of cranial cruciate ligamentdeficient stifle with the fabelo-tibial suture technique. The instantaneous center of motion and resulting velocity vectors were determined by radiographic examination of the stifle joint in twelve dogs before and after cranial cruciate ligament transection, and after joint stabilization. All the stifles showed normally positioned instantaneous centers of motion and velocity vectors before cranial cruciate ligament transection. Eleven joints had abnormally positioned velocity vectors after transection of the cranial cruciate ligament. After joint stabilization, four joints in the stainless steel wire suture group continued showing abnormal velocity vectors, while all stifles joints in the polypropylene group had normal positioned velocity vectors. The polypropylene suture is more adequate for extra-articular stabilization as it maintains the normal joint biomechanics.

Key words: surgery, biomechanics, orthopedics.

\section{INTRODUÇÃO}

As artropatias envolvendo o joelho estão entre as moléstias mais freqüentemente diagnosticadas na prática clínico-cirúrgica de pequenos animais, sendo a ruptura do ligamento cruzado cranial (LCCr) causa comum de claudicação dos membros pélvicos (PAYNE \& CONSTANTINESCU, 1993; DECAMP et al., 1996; JACKSON et al., 2001).

Em Medicina Veterinária, a análise do centro instantâneo do movimento (CIM) tem se mostrado um método clinicamente eficiente e simples para se analisar a biomecânica da articulação do joelho, promovendo a interpretação objetiva do grau de estabilidade articular (ARNOCZKY et al., 1977; IRELAND et al., 1986; MITTON et al., 1991; PROSTREDNY et al., 1991).

O CIM é determinado como um ponto localizado no fêmur, em qualquer fase de movimento

\footnotetext{
${ }^{1}$ Médico Veterinário. Mestre, Professor Substituto, Universidade de Brasília (UnB), Faculdade de Agronomia e Medicina Veterinária (FAV), CP 04508, 70910-970, Brasília-DF. E mail: selmi@unb.br Autor para correspondência.

${ }^{2}$ Acadêmico, Curso de Graduação em Medicina Veterinária, FAV-UnB.
} 
deste osso em relação à tíbia, no qual a velocidade do vetor é igual a zero. As posições normais para o CIM na articulação intacta do joelho em cães já foram determinadas previamente e não foram encontradas diferenças significativas entre as articulações dos membros direito e esquerdo, ou entre cães vivos e cadáveres (IRELAND et al., 1986). A articulação normal do joelho possui movimentos de deslizamento e rolagem. Se o CIM estiver situado em um ponto em contato com a superfície articular do fêmur e tíbia, estará ocorrendo predomi-nantemente um movimento de rolagem entre as duas epífises ósseas. Se o CIM encontrar-se em uma posição extra-articular estará ocorrendo predomi-nantemente um movimento de deslizamento entre as duas extremidades articulares. (FRANKEL et al., 1971; ARNOCZKY et al., 1977; IRELAND et al., 1986; PROSTREDNY et al., 1991).

$\mathrm{O}$ vetor velocidade, que determina as forças compressivas existentes na articulação, é representado por uma reta tangente à superfície articular quando as forças atuantes na articulação apresentam-se neutralizadas; desta forma, propiciando menor resistência ao movimento de flexão e extensão do membro. (PROSTREDNY et al., 1991). Essa condição é observada, quando o CIM encontra-se posicionado na superfície articular no ponto de contato entre as cartilagens articulares, ou quando está localizado de forma extra-articular, perpendicularmente ao ponto de contato articular. Se o CIM localiza-se em uma linha não perpendicular ao ponto de maior contato das superfícies articulares, o vetor velocidade naquele ponto não será tangente à superfície condilar, resultando em forças compressivas nas superfícies articulares e conseqüentemente no aumento das forças de fricção durante a movimentação do membro (FRANKEL et al., 1971; ARNOCZKY et al., 1977; PROSTREDNY et al., 1991).

Existem várias técnicas cirúrgicas para a estabilização da ruptura do LCCr, e estas são divididas tradicionalmente em reparos extra e intraarticulares. As técnicas intra-articulares utilizam material autógeno, alógeno ou sintético para conferir estabilidade funcional da articulação (DENNY \& GOODSHIP, 1980), enquanto as técnicas extra-articulares alteram as relações entre as estruturas anatômicas peri-articulares, mimetizando a restrição estabelecida pelo $\mathrm{LCCr}$ intacto (DE ANGELIS \& LAU, 1970; SMITH \& TORG, 1985). todos os procedimentos que resultam em biomecânica eficiente, podendo assim acelerar a doença articular degenerativa (ARNOCZKY et al., 1977).

A sutura fabelo-tibial é a técnica extraarticular mais comumente usada, e tem se mostrado bastante eficiente na estabilização da articulação do joelho após ruptura do LCCr (KORVICK et al., 1994). Alguns relatos sugerem sua superioridade em relação a outras técnicas (MOORE \& READ, 1995), principalmente por ser um procedimento menos traumático que a estabilização intra-articular e por promover um melhor comportamento da biomecânica imediatamente após a cirurgia (PATTERSON et al., 1991; OLMSTEAD, 1993). Um estudo clínico avaliando animais submetidos a essa técnica por um período de 9 a 34 meses após a cirurgia revelou que $86 \%$ dos animais estavam clinicamente perfeitos ou apresentavam dor intermitente no membro afetado (MOORE \& READ, 1996).

No passado, a sutura fabelo-tibial só era utilizada na estabilização articular de animais com menos de 15 ou $20 \mathrm{~kg}$, porém, o uso de fio de aço na estabilização extra-articular após ruptura do LCCr tem se mostrado bastante efetivo na estabilização articular de cães de raças de grande porte (OLMSTEAD, 1993).

Os materiais de sutura comumente usados para o reparo extra-articular do LCCr, como o poliéster multifilamentar e aço, são relativamente inelásticos e evitam a rotação axial normal necessária para o movimento correto da articulação do joelho, enquanto a utilização de suturas com propriedades mais elásticas como o polibutéster monofilamentar pode preservar a mecânica normal da articulação do joelho (PROSTREDNY et al., 1991).

Um estudo prévio indicou que as técnicas intra-articulares foram bem sucedidas na manutenção da biomecânica normal da articulação do joelho através da determinação pelo CIM, e que a técnica extra-articular de posicionamento da sutura entre a fabela lateral e o tendão patelar distal causou mudança do vetor velocidade para uma posição anormal. Foi sugerida a hipótese de que o tensionamento das estruturas extra-articulares restrinja a rotação axial normal da tíbia, resultando em movimento articular anormal (ARNOCZKY et al., 1977).

O objetivo deste estudo foi analisar os efeitos na biomecânica articular de dois materiais de sutura, fio de aço e fio de polipropileno, através da avaliação ex-vivo do centro instantâneo de movimento e análise do vetor velocidade, após estabilização extra-articular por meio da sutura fabelo-tibial em cães. 
Existem divergências consideráveis em relação à superioridade destas técnicas quanto ao melhor retorno da função e quanto à estabilidade articular. Alguns relatos têm sugerido que não são MATERIAL E MÉTODOS

Foram utilizados 12 membros pélvicos caninos, de cadáveres com peso entre 7 e $17 \mathrm{~kg}$, provenientes do canil do Centro de Controle de Zoonoses do Distrito Federal (CCZ-DF). Os membros pélvicos foram aleatoriamente divididos em dois grupos de seis componentes cada. No primeiro grupo, utilizou-se fio de aço monofilamentara número 2 (grupo 1) e no segundo grupo, fio de polipropileno ${ }^{\mathrm{b}}$ número 2 (grupo 2).

Foram realizadas radiografias prévias para determinação da normalidade da articulação fêmurtíbio-patelar, com incidências médio-laterais, posicionando-se a articulação paralela ao filme radiográfico em um ângulo de $90^{\circ}$ de flexão e em extensão total. As projeções foram realizadas com o membro apoiado diretamente sobre o chassi a uma distância foco-filme de aproximadamente $100 \mathrm{~cm}$. O plano da flexão articular foi paralelo ao plano do filme, com o raio centrado na articulação. Após a primeira série de radiografias, foi realizada a secção do ligamento cruzado cranial, através de pequena incisão percutânea medial, com lâmina de bisturi introduzida em direção crânio-caudal paralelamente aos côndilos tibiais. O movimento de gaveta cranial e a excessiva rotação interna da tíbia foram observados à palpação e, posteriormente, uma segunda série de radiografias foi realizada, obedecendo aos mesmos critérios de posicionamento previamente descritos.

Após estes procedimentos, os membros foram posicionados em decúbito dorsal. Realizou-se artrotomia exploratória parapatelar lateral para confirmação da transecção completa do ligamento cruzado cranial e integridade do ligamento cruzado caudal, sendo que as porções remanescentes do $\mathrm{LCCr}$ foram removidas. A cápsula articular foi suturada utilizando-se um padrão interrompido simples com Poliglactina $910^{c}$ número 3-0. Em seguida, foi realizada a estabilização extra-articular através da técnica de sutura fabelo-tibial, conforme descrição de DE ANGELIS \& LAU (1970). Novas avaliações radiográficas foram realizadas, todas em um período que não ultrapassasse 60 minutos após a eutanásia, com o intuito de se evitar os efeitos indesejáveis do "rigor mortis".

O CIM e o vetor velocidade resultante foram calculados antes da transecção do ligamento cruzado cranial; após a transecção do ligamento; e após estabilização cirúrgica, em cada série de duas sucessivas radiografias. Para tanto, utilizou-se o método de "Rouleaux", que consistiu na sobreposição das tíbias nas projeções em flexão e extensão e na determinação de dois pontos de referência idênticos, localizados nos fêmures, com intervalo mínimo de distância de 4 a $5 \mathrm{~cm}$ entre eles. O deslocamento respectivo dos dois pontos demarcados no fêmur foi unido por uma reta, e posteriormente, determinou-se as bissetrizes a essas linhas, sendo que a intersecção destas determinou o CIM. O CIM foi então unido por uma reta ao ponto de maior contato articular, determinando assim o eixo CIMp (Figura 1). Para determinação do vetor velocidade resultante, uma reta foi traçada perpendicularmente à CIMp, conforme sugeriram FRANKEL et al. (1971) e PROSTREDNY et al. (1991) (Figura 1).

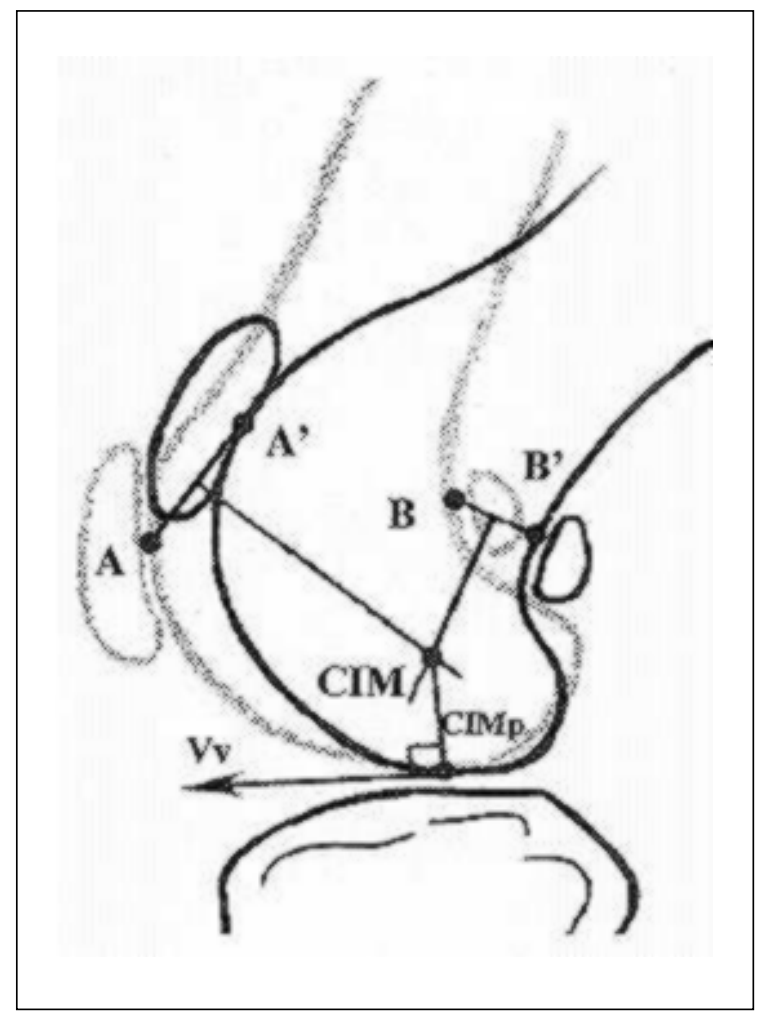

Figura 1 - Esquema para determinação do centro instantâneo de movimento (CIM) na articulação fêmur-tíbio-patelar. Notar a sobreposição da tíbia com deslocamento femoral, onde se identificam os pontos A e B, e seus similares, A' e B'. As bissetrizes dos segmentos A-A' e B-B' determinam o CIM. O CIM é então unido por um segmento de reta (CIMp) ao ponto de maior contato articular. O vetor velocidade $(\mathrm{Vv})$ é obtido a partir da perpendicular a CIMp.

Ciência Rural, v. 33, n. 5, set-out, 2003. 


\section{RESULTADOS E DISCUSSÃO}

Durante a avaliação física prévia de todos membros pélvicos utilizados, foi constatada ausência de movimento de gaveta e quaisquer alterações músculo-esqueléticas. No momento inicial, todas articulações do grupo 1 e grupo 2 apresentavam o CIM extra-articular com posição centrada no côndilo femoral, com predominância de um movimento de deslizamento da articulação (ARNOZCKY et al., 1977; IRELAND et al., 1986; PROSTREDNY et al., 1991). Todos os membros dos grupos 1 e 2 apresentaram normalidade do vetor velocidade que era tangente à superfície articular antes da transecção do LCCr (Tabela 1).

Após transecção do LCCr, observou-se sinal de gaveta positivo em todas articulações. Durante a artrotomia e avaliação radiográfica, não foram evidenciados sinais de doença articular prévia. Também não foram observados sinais de lesão iatrogênica ao ligamento cruzado caudal. A avaliação posterior à secção do ligamento cruzado cranial revelou que cinco articulações de cada grupo mantiveram o CIM em posição extra-articular, enquanto que duas articulações (um cão de cada grupo) apresentaram o CIM em posição intra-articular. Todos os membros pélvicos do grupo 1 e cinco do grupo 2 apresentaram o vetor velocidade deslocado para uma posição anormal com aumento das forças compressivas atuantes na articulação, responsáveis pela degeneração articular progressiva (ARNOCSKY et al., 1977). Um único membro do grupo 2 continuou apresentando o vetor velocidade normal, com posição tangencial à superfície articular, que pode ter decorrido de erro na determinação do ponto de maior contato entre o fêmur e a tíbia ou de um posicionamento neutro durante a avaliação radiográfica (FRANKEL et al., 1971; GERBER \& MATTER, 1983; IRELAND et al., 1986; PROSTREDNY et al., 1991).

Após a estabilização articular, foi constatada a ausência de movimento de gaveta cranial, comprovando a efetividade clínica da estabilização. Radiograficamente, observou-se que uma articulação do grupo 1 apresentou deslocamento do CIM para uma posição intra-articular, enquanto as outras articulações deste grupo mantiveram o CIM extra-articular. Duas articulações exibiam o vetor velocidade tangente à superfície articular, porém com leve deslocamento quando comparado aos momentos anteriores a transecção do LCCr, enquanto quatro articulações apresentaram o vetor em posição deslocada, demonstrando desta forma que o fio de aço altera a dinâmica articular, possivelmente em virtude de suas propriedades inelásticas.

No grupo 2, um membro voltou a apresentar o CIM extra-articular, semelhante aos outros animais. Todas as articulações evidenciaram posicionamento do vetor velocidade tangente à superfície articular,

Tabela 1 - Valores obtidos durante avaliação ex-vivo do centro instantâneo de movimento (CIM), vetor velocidade (VETOR) e teste do movimento de gaveta (TGAVE) de articulações fêmur-tíbio-patelares submetidas a transeç̧ão do ligamento cruzado cranial e estabilizadas por meio de sutura fabelo-tibial com emprego de fio de aço inoxidável (grupo 1) ou fio de polipropileno (grupo 2).

GRUPO 1 - Aço inoxidável

\begin{tabular}{|c|c|c|c|c|c|c|c|c|c|c|c|c|c|c|c|c|c|c|}
\hline & \multicolumn{18}{|c|}{ ANIMAL } \\
\hline & \multicolumn{3}{|c|}{1} & \multicolumn{3}{|c|}{2} & \multicolumn{3}{|c|}{3} & \multicolumn{3}{|c|}{4} & \multicolumn{3}{|c|}{5} & \multicolumn{3}{|c|}{6} \\
\hline & T1 & $\mathbf{T 2}$ & T3 & T1 & $\mathbf{T 2}$ & T3 & $\mathbf{T 1}$ & $\mathbf{T} 2$ & T3 & T1 & $\mathbf{T 2}$ & T3 & T1 & $\mathbf{T 2}$ & T3 & $\mathbf{T 1}$ & $\mathbf{T 2}$ & T3 \\
\hline CIM & $\mathrm{E}$ & $E$ & $E$ & $E$ & $E$ & I & $\mathrm{E}$ & $\mathrm{E}$ & E & $E$ & $\mathrm{E}$ & $\mathrm{E}$ & $\mathrm{E}$ & $E$ & $E$ & $\mathrm{E}$ & I & $\mathrm{E}$ \\
\hline VETOR & $\mathrm{N}$ & A & $\mathrm{N}$ & $\mathrm{N}$ & A & A & $\mathrm{N}$ & A & $\mathrm{N}$ & $\mathrm{N}$ & A & A & $\mathrm{N}$ & A & A & $\mathrm{N}$ & A & A \\
\hline TGAVE & - & + & - & - & + & - & - & + & - & - & + & - & - & + & - & - & + & - \\
\hline \multicolumn{19}{|c|}{ GRUPO 2 - Polipropileno } \\
\hline & \multicolumn{18}{|c|}{ ANIMAL } \\
\hline & & 1 & & & 2 & & & 3 & & & 4 & & & 5 & & & 6 & \\
\hline & T1 & T2 & T3 & T1 & T2 & T3 & T1 & $\mathbf{T} 2$ & T3 & T1 & $\mathbf{T 2}$ & T3 & T1 & $\mathbf{T 2}$ & T3 & T1 & $\mathbf{T} 2$ & T3 \\
\hline CIM & $\mathrm{E}$ & I & $\mathrm{E}$ & $\mathrm{E}$ & $\mathrm{E}$ & I & $\mathrm{E}$ & $\mathrm{E}$ & $\mathrm{E}$ & $\mathrm{E}$ & E & $\mathrm{E}$ & E & E & E & $\mathrm{E}$ & E & $\mathrm{E}$ \\
\hline VETOR & $\mathrm{N}$ & A & $\mathrm{N}$ & $\mathrm{N}$ & A & $\mathrm{N}$ & $\mathrm{N}$ & A & $\mathrm{N}$ & $\mathrm{N}$ & A & $\mathrm{N}$ & $\mathrm{N}$ & $\mathrm{N}$ & $\mathrm{N}$ & $\mathrm{N}$ & A & $\mathrm{N}$ \\
\hline TGAVE & - & + & - & - & + & - & - & + & - & - & + & - & - & + & - & - & + & - \\
\hline
\end{tabular}

E: Extra-articular; I:Intra-articular; N: Normal; A: Anormal; -: Negativo; +: Positivo T1: Momento antes da transecção do LCCr; T2: Momento após transecção do LCCr; T3: Momento após estabilização articular. 
comprovando uma biomecânica articular normal, provavelmente decorrente das propriedades elásticas do fio de polipropileno, o que permitiu adequado movimento de flexão e extensão articular, acompanhado de rotação interna da tíbia. Em um cão, apesar do vetor ter posicionamento tangente à superfície articular, este apresentava posicionamento discretamente alterado quando comparado ao momento anterior a transecção do LCCr.

Um animal do grupo 2 apresentou ainda, subluxação cranial da tíbia nas radiografias, posteriormente à ruptura e à estabilização do $\mathrm{LCCr}$, apesar de uma localização correta do CIM e do vetor velocidade. Uma segunda articulação do grupo 2 apresentou deslocamento do CIM de posição extraarticular para posição intra-articular; entretanto, não se observou alteração do vetor velocidade, sugerindo que a articulação parou de exercer movimento de deslizamento e adquiriu movimento de rolagem. A manutenção do sentido do vetor velocidade, neste caso, indicou que forças compressivas não atuavam na articulação, o que implica em menor atrito entre as superfícies articulares do fêmur e tíbia. (FRANKEL et al., 1971; ARNOCZKY et al., 1977; IRELAND et al., 1986; PROSTREDNY et al., 1991). As observações pré, trans e pós-operatórias relacionadas ao CIM, vetor velocidade e presença do movimento de gaveta estão dispostas na tabela 1 .

Existem diversas técnicas para estabilização articular após ruptura do ligamento cruzado cranial, porém a maioria destes procedimentos parece produzir resultados similares e insatisfatórios com relação à manutenção da biomecânica articular normal (PATTERSON et al., 1991; PROSTREDNY et al., 1991). Com base nos resultados desta pesquisa, a estabilização por meio da técnica extra-articular fabelo-tibial mostrou-se eficiente após transecção do ligamento cruzado cranial. A superioridade do fio de polipropileno, quando comparado ao aço, deve ser atribuída às suas propriedades elásticas gerando, conseqüentemente, maior adaptabilidade à dinâmica articular, fator que o torna uma alternativa viável na tentativa de se retardar a progressão da doença articular degenerativa (ARNOCZKY et al., 1977; HENON, 1996). Apesar de não evitar a progressão da doença articular degenerativa, o uso do fio de aço constitui uma alternativa na estabilização da ruptura do $\mathrm{LCCr}$ em cães de grande porte (OLMSTEAD, 1993). No entanto, de acordo com os achados deste estudo, é recomendável sua substituição pelo fio de polipropileno, já que a manutenção da normalidade do vetor velocidade foi superior quando comparada à do fio de aço, contrariamente as citações de PROSTREDNY et al. (1991), que sugeriram que o uso da técnica fabelo-tibial não demonstra alteração da biomecânica articular em função do material de sutura empregado.

A análise do centro instantâneo do movimento mostrou-se eficiente na determinação do grau de estabilização e alteração da biomecânica articular, principalmente por ser um método de fácil aplicação e por seus resultados serem reproduzíveis devido à sua objetividade (ARNOCSKY et al., 1977; PROSTREDNY et al., 1991), porém, é importante ressaltar a dificuldade do posicionamento correto do membro durante a exposição radiográfica (os côndilos femorais devem estar paralelos com sobreposição condilar) e da determinação do ponto de contato articular, pois não existe relato na literatura sobre sua exata localização e, conforme observado, trata-se de uma superfície de contato, e não de um único ponto. Outrossim, durante a avaliação radiográfica, apesar do paralelismo condilar necessário, os efeitos dos movimentos rotacionais da tíbia são negligenciados. Contudo, este parece ser um meio prático de se obter informações sobre a biomecânica dos joelhos normais e anormais.

A determinação do vetor velocidade observada em posição normal em um animal, após a ruptura do ligamento, pode ser decorrente de uma posição neutra no momento da radiografia, sugerindo que a radiografia sob estresse possa representar uma alternativa para se aumentar a eficiência na determinação do CIM e do vetor velocidade, no diagnóstico da ruptura do ligamento cruzado cranial. Desta forma, o deslocamento cranial da tíbia e conseqüente alteração de posicionamento do vetor velocidade seriam intensificados.

Apesar da variação de pesos encontrados entre os animais deste estudo, não foram observadas dificuldades nas determinações do CIM e vetor velocidade, assim como descrito por PROSTREDNY et al., (1991), os quais referiram que o tamanho dos animais não é fator negativo na determinação dos pontos de referência femorais.

A prevalência de um CIM extra-articular repetiu os resultados obtidos em estudos anteriores, refletindo o movimento predominante de deslizamento da articulação do joelho canino (ARNOCSKY et al., 1977; PROSTREDNY et al., 1991). Porém, a mudança da direção do vetor velocidade após estabilização articular foi observada em quatro animais do grupo 1, demonstrando alteração da biomecânica articular, relatada antes somente em humanos após a ruptura do LCCr, contradizendo os resultados obtidos 
anteriormente em cães (FRANKEL et al., 1971; GERBER \& MATTER, 1983; PROSTREDNY et al., 1991). As diferenças nos resultados podem ser decorrentes do posicionamento dos pontos de ancoragem através de uma perfuração na crista da tíbia, semelhantes a PROSTREDNY et al., (1991), resultando em menor extensão da articulação do joelho (ARNOZCKY et al., 1977; PROSTREDNY et al., 1991). Entretanto, há de se considerar que a localização da perfuração tibial possa ter influencia sobre a biomecânica articular, uma vez que, quanto mais caudal esta for realizada, menor será a extensão possibilitada pela sutura fabelo-tibial lateral, sugerindo que estudos sejam realizados para avaliar o real efeito da posição da perfuração tibial sobre o CIM e o vetor velocidade resultante, em cães.

Uma vez que a determinação do CIM e do vetor velocidade indicam o movimento predominante na articulação, há de se considerar que este método possa ser um auxílio importante na determinação do prognóstico após estabilização cirúrgica.

\section{CONCLUSÃO}

A determinação do centro instantâneo do movimento é uma alternativa adequada para avaliação da biomecânica articular após a estabilização ex-vivo da ruptura do ligamento cruzado cranial por meio de sutura fabelo-tibial em cães. A técnica extra-articular utilizando fio de polipropileno, mostra-se eficaz na restituição da estabilidade do joelho após transecção desse ligamento, principalmente por não alterar a biomecânica normal dessa articulação.

\section{FONTES DE AQUISIÇÃO:}

a - ciflex $^{\circledR}$ : Ethicon, Brasil

b - Prolene ${ }^{\circledR}$ : Ethicon, Brasil.

c - Vycril ${ }^{\circledR}$ : Ethicon, Brasil.

\section{REFERÊNCIAS BIBLIOGRÁFICAS}

ARNOCZKY, S.P.; TORZILLI, P.A.; MARSHALL, J,L. Biomechanical evaluation of anterior cruciate ligament repair in the dog: An analysis of the ICM. J Am Anim Hosp Assoc, v.13, p.553-558, 1977.

DE ANGELIS, M.; LAU, R.E. A lateral retinacular imbrication techynique for the surgical correction of anterior cruciate ligament rupture in the dog. J Am Vet Med Assoc, v.157, p.79-84, 1970.

DECAMP, C.E. et al. Kinematic evaluation of gait in dogs with cranial cruciate ligament rupture. Am J Vet Res, v.57, n.1, p.120126, 1996.
DENNY, H.R.; GOODSHIP, A.E. Replacement of the anterior cruciate ligament with carbon fibre in the dog. J Small Anim Pract, v.21, p.279-286, 1980.

FRANKEL, V.H.; BURSTEIN, A.H.; BROOKS, D.B. Biomechanics of internal derangement of the knee. Pathomechanics as determined by analysis of the ICM. J Bone Joint Surg, v.53A, p.945-962, 1971.

GERBER, C.; MATTER, P. Biomechanical analysis of the knee after rupture of the anterior cruciate ligament and its primary repair. An instant-centre analysis of function. J Bone Joint Surg, v. 65, p. 391-399, 1983.

HENON, M.R. Stifle joint. In: LIPOWITZ, A.J. et al. Complications in small animal surgery. Baltimore : Willians \& Wilkins, 1996. Cap.21, p.621-630.

IRELAND, W.P.; ROGERS, J.; MYERS, R.K. Location of the instantaneous center of rotation in the normal canine stifle. Am J Vet Res, v.47, p.837-840, 1986.

JACKSON, J. et al. Pathologic changes in grossly normal menisci in dogs with rupture of the cranial cruciate ligament. J Am Vet Med Assoc, v.218, n.8, p.1281-1284, 2001.

KORVIKC, D.L.; JOHNSON, A.L.; SCHAEFFER, D.J. Surgeon's preferences in treating cranial cruciate ligament ruptures in dogs. J Am Vet Med Assoc, v.205, n.9, p.1318-1324, 1994.

MITTON, G.R.; IRELAND, W.P.; RUNYON, C.L. Evaluation of the instantaneous center of rotation of the stifle before and after repair of torn cruciate ligament by use of the over-the-top technique in dogs. Am J Vet Res, v.52, n.10, p.1731-1737, 1991.

MOORE, K.W.; READ, R.A. Cranial cruciate ligament rupture in the dog - a retrospective study comparing surgery techniques. Aust Vet J, v.72, n.8, p.281-285, 1995.

MOORE, K.W.; READ, R.A. Rupture of the cranial cruciate ligament in dogs-part II. Comp Cont Educ Prac Vet, v.18, n.4, p.381-391, 1996.

OLMSTEAD, M.L. The use of orthopedic wire as a lateral suture for stifle stabilization. Vet Clin North Am: Small Anim Pract, v.23, n.4, p.735-753, 1993.

PATTERSON, R.H. et al. Biomechanical stability of four cranial cruciate ligament repair techniques in the dog. Vet Surg, v.20, n.2, p.85-90, 1991

PAYNE, J.T.; CONSTANTINESCU, G.M. Stifle joint anatomy and surgical approaches in the dog. Vet Clin North Am: Small Anim Pract, v.23, n.4, p.691-701, 1993.

PROSTREDNY, J.M. et al. Effect of suture type on stifle joint biomechanics after extra-articular repair of cranial cruciate ligament transection in the dog. Vet Comp Orthop Traumatol, v.4, p.144-149, 1991.

SMITH, G.K.; TORG, J.S. Fibular head transposition for repair of cruciate-deficient stifle in the dog. J Am Vet Med Assoc, v.187, p. 375-383, 1985. 